\title{
LA PROFILAXIS DEL VIENTO. INSTITUCIONES REPRESIVAS Y SANITARIAS EN LA PATAGONIA ARGENTINA, 1880-1940*
}

\author{
Ernesto Bohoslavsky \\ Universidad Nacional de General Sarmiento
}

María Silvia Di Liscia

Universidad Nacional de La Pampa

\section{RESUMEN}

Este artículo avanza sobre el significado de las tecnologías y discursos del denominado «control social» en el interior argentino, haciendo hincapié en los Territorios Nacionales de La Pampa, Neuquén y Río Negro, entre 1880-1940. En esos espacios, de jurisdicción federal, se tomará en cuenta la conformación de instituciones represivas como policía y cárceles y sanitarias, como hospitales y asilos, así como la aplicación de metodologías positivistas para el estudio y el establecimiento de grillas clasificatorias de demarcación de la anormalidad. Una dependencia mayor del Estado nacional podría haber significado atención al desarrollo económico y social, a partir de las instituciones centrales para el proyecto modernizador y positivista. Pero un examen más profundo de las formas y los procesos históricos, que incluyen las dificultades para su implementación en su formación, el sostén presupuestario y los problemas permanentes de funcionamiento, implica una revisión de las capacidades de acción y las posibilidades reales de establecimiento de un programa general de control social en Argentina, como han sostenido muchos historiadores concentrados en el caso de Buenos Aires.

PALABRAS CLAVE: Argentina. 1880-1940. Control social. Territorios Nacionales. Salud.

\section{THE WIND PROPHYLAXIS. REPRESSIVE AND SANITARY INSTITUTIONS IN ARGENTINA'S PATAGONIA, 1880-1940}

\section{ABSTRACT}

This article focuses on the study of some social control technologies and discourses, displayed in Argentina's provinces between 1880 and 1940, with particular reference to the so-called 'Terri-

* Este artículo es parte del proyecto PICTO 18-30782 (Directorio ANPC y $\operatorname{Tn}^{\circ}$ 092/06), UNLPam y del proyecto HUM2005-03777 (Plan Nacional de Investigaciones I+D, Secretaría de C y T, Ministerio de Educación y Ciencia, España) 
torios Nacionales' of La Pampa, Río Negro and Neuquén, which were submitted to a direct federal authority. The main purpose is to analyze - within these areas - the building of repressive and sanitary institutions (i. e., police, prisons, asylums, hospitals) as well as the enforcement of positivists studying and classifying methodologies, intended to identify 'abnormality'. A straight and permanent rule of these 'Territorios Nacionales' on the federal State could have meant a longer attention to their social and economic development through a direct and intense presence of national, modernizing, positivist institutions. However, a deeper historical study of repressive and sanitary institutions allows to arrive to completely different conclusions. The sources show that these institutions had numerous daily problems, were frequently and severely under-budgeted, and were obliged to develop not originally foreseen functions and tasks. These situations imply revising not only these institutions' real regulation capacities but also the very existence of a generalized, efficient social control programme in Argentina at the beginning of the $20^{\text {th }}$ century, as many scholars focused on Buenos Aires's study case have already argued.

KEY WORDS: Argentina. 1880-1940. Social control. Territorios Nacionales. Health system.

En 1917 el comisario inspector de policía del cordillerano Territorio Nacional de Neuquén, Lucrecio Gómez, elevó al gobernador un informe después de su inspección de la zona norte del Territorio ${ }^{1}$. En ese informe, Gómez hacía llegar distintas sugerencias y solicitudes acerca de la paupérrima situación en que se encontraban los edificios policiales. Entre otras cosas, le requería al gobernador el envío de chapas de zinc para refaccionar las comisarías, de manera tal de evitar que se siguieran inundando las sedes policiales cada vez que llovía «al extremo de imposibilitar a veces el trabajo de las oficinas y evitar también las continuas enfermedades del personal». Pero también planteaba algunas necesidades de la región sobre la que acababa de realizar la inspección, entre las cuales destacaba el envío de un médico público dado que «los innumerables enfermos que a veces suelen existir se ven en la tristísima necesidad de recurrir a los curanderos y curanderas que, por lo general, ocasionan la muerte de los enfermos».

De la exposición del comisario inspector se desprende una imagen que este artículo pretende dar por cierta: en las áreas pampeano-patagónicas, era muy marcada la precariedad de la infraestructura pública (y la de salud en particular), lo cual permitió la supervivencia de las prácticas populares de sanación frente al avance de las instituciones y agentes de la medicina "científica», mucho más exitosas en regiones centrales de Argentina en el medio siglo posterior a 1880. En efecto, la realidad que muestra el testimonio del comisario Gómez parece desdecir esas miradas más generales de la historiografía argentina sobre el proceso de medicalización y la universalización de las

1 Sepúlveda, P.R. (1991), Libro de Oro de la Policía, Neuquén, Círculo Policial Neuquino, pp. 136 y ss. 
prácticas sanitarias lideradas y/o legitimadas por el Estado ${ }^{2}$. En lugar de descubrir el «Estado médico-legal» que han señalado algunos autores para la Argentina de principios de siglo $^{3}$, una revisión de la historia de la mitad sur del país evidencia que las instituciones de salud mostraban claramente un bajo nivel de desarrollo y de capacidades para intervenir sobre la vida y cuerpos de los habitantes. De acuerdo a ideas que hemos expuesto recientemente ${ }^{4}$, estudiar a las instituciones públicas de las regiones pampeano-patagónicas permite complejizar la historia de la salud y del control social en Argentina en varios sentidos: señalar cuáles fueron los límites materiales y humanos de las políticas públicas e indicar la falta de coherencia y sistematicidad de esas políticas, en las cuales lo contingente y las soluciones temporales tenían un peso que no siempre ha sido reconocido por la literatura.

Este artículo pretende dar cuenta de la naturaleza de las intervenciones en salud desarrolladas en los Territorios Nacionales del sur argentino entre el momento de su creación en la década de 1880 y los prolegómenos del fenómeno peronista, en la década de 1940. Para ello, primero se ofrece una caracterización de esas tierras «nuevas» y de su ocupación y puesta en producción tras el sometimiento de las sociedades indígenas. A continuación, se exponen las principales características del, por así decir, «sistema de salud» de esa enorme región y luego, se hace hincapié en los servicios de salud existentes dentro de instituciones como cárceles y escuelas. A partir de documentos de variado origen (registros oficiales del Departamento Nacional de Higiene, del Consejo Nacional de Educación y del Ministerio de Justicia e Instrucción, Memorias de los Gobernadores, informes de los directores de cárceles y de la prensa científica y de divulgación, entre otros $)^{5}$, las conclusiones de este tra-

2 Un esquema general en Porter, D. (ed.) (1994), The History of Public Health and the Modern State, Atlanta, Clio Medica; para Argentina, ver ARMus, D. (2000), El descubrimiento de la enfermedad como problema social. En LoBATo, M.Z. (dir.), Nueva Historia Argentina, T. V: El progreso, la modernización y sus límites (1880-1916), Buenos Aires, Sudamericana, pp. 507-551; un análisis general en Di LiSCIA, M.S. (2008), Reflexiones sobre la 'nueva historia social' de la salud y la enfermedad en Argentina. En CARBONETTI, A. y GonZÁLEZ LEANDRI, R. (eds.), Historia de la salud y la enfermedad, Córdoba, UNC, pp. 15-47.

3 Salvatore, R. (2001), Sobre el surgimiento del Estado Médico legal en la Argentina, (1890-1940), Estudios Sociales, XI (20), 81-114 y RodRíGUEZ, J. (2006), Civilizing Argentina. Science, Medicine and The Modern State, Chapell Hill, University of North Carolina Press.

4 (2005), Introducción. Para desatar algunos nudos (y atar otros). En Di Liscia, M.S. y Bohoslavsky, E. (comp.), Instituciones y formas de control social en América Latina, 18401940. Una revisión, Buenos Aires, EDULPAM-UNGS-Prometeo Ediciones, pp. 9-19.

5 La documentación se encuentra en: Archivo y Biblioteca de la Facultad de Medicina (UBA, Buenos Aires), Archivo General de la Nación (Ministerio del Interior y Ministerio de 
bajo desean contribuir a que se perciba con mayor profundidad la diversidad de las prácticas de curación y de intervención en el territorio argentino.

\section{LAS TIERRAS «NUEVAS» Y LOS RASGOS DEL ESTADO REALMENTE EXISTENTE}

La ocupación por parte del Ejército de línea de los territorios al sur de Buenos Aires en 1879 permitió que el Estado argentino incorporara a su soberanía millones de hectáreas, y que extendiera sus límites internacionales hasta la Cordillera de los Andes y el extremo austral. En los años posteriores las «tierras nuevas» fueron en su mayoría rematadas, vendidas a particulares o entregadas como premio a los militares que habían participado de la campaña. El destino económico en las tierras pampeanas y patagónicas tras su ocupación distó de ser homogéneo: hacia 1900 coexistían áreas de pleno desarrollo del capitalismo rural con otras en las que sobrevivían prácticas productivas y comerciales de más profunda raigambre en la vida fronteriza. Pero también variaban las estrategias productivas y los bienes producidos: enormes latifundios dedicados a los ovinos en Santa Cruz y Chubut coexistían con los sistemas de arrendamientos en La Pampa oriental, monoproductores cerealeros. Las zonas cordilleranas de Neuquén y el sur de Río Negro contenían reservas de indígenas y pastores trashumantes, dueños de unas pocas cabras, viviendo en un marco de profunda precariedad material y aislamiento geográfico, condiciones que no se han visto alteradas en demasía en la actualidad.

La diversidad de espacios productivos se expresó en muy distintos patrones de relación laboral y de residencia, modificando los niveles de concentración demográfica en el área pampeano-patagónica (Cuadro $n^{0} 1$ ). Así, las regiones australes tuvieron a lo largo la primera mitad del siglo XX una muy débil densidad demográfica, resultado del predominio de los latifundios ovinos atendidos por una mano de obra escasa, convocada para el período de la esquila. El este de la Pampa y valles de Río Negro, por el contrario, mostraron un notorio crecimiento poblacional por el aluvión de migrantes de otras provincias y de ultramar, deseosos de incorporarse a áreas capitalistas de la agricultura, sea como peones, arrendatarios o en actividades secundarias y terciarias de apoyo (agro-industria, bancos, comercios, ferrocarril, etc.). Hacia 1920, cierta densidad poblacional se evidenciaba en algunas ciudades con miles de habitan-

Relaciones Exteriores), Biblioteca del Maestro (Palacio Pizzurno: Ministerio de Educación), Archivo Histórico Provincial de Neuquén, Archivo Histórico Provincial de La Pampa y Archivo de la Justicia Letrada del Territorio de Neuquén. 
tes, dotadas de una animada vida social, mutualista, comercial y por momentos, política. El asociacionismo y el voluntarismo local permitían atemperar ausencias o debilidades del sector público, especialmente en áreas como la salud, la educación, la infraestructura urbana y el entretenimiento.

CUADRO N ${ }^{\circ}$ 1. POBLACIÓN Y SUPERFICIE DE LOS TERRITORIOS NACIONALES, $1895-1936^{6}$

\begin{tabular}{|l|l|c|r|r|r|}
\hline & $\begin{array}{l}\text { Territorios } \\
\text { Nacionales }\end{array}$ & $\begin{array}{c}\text { Superficie } \\
\left(\mathbf{k m}^{2}\right)\end{array}$ & \multicolumn{3}{|c|}{ Población } \\
$\mathbf{1 9 1 4}$ & \multicolumn{1}{c|}{$\mathbf{1 9 3 6}^{* *}$} \\
\hline & Misiones & 29.229 & 33.163 & 53.563 & 168.831 \\
\hline & Formosa & 107.258 & 4.829 & 19.281 & 49.634 \\
\hline & Chaco & 136.635 & 10.422 & 46.274 & 270.439 \\
\hline & Los Andes & $90.644^{*}$ & - & 2.487 & 6.810 \\
\hline \multirow{4}{*}{\begin{tabular}{l} 
Área $\begin{array}{l}\text { Pampeano- } \\
\text { Patagónica }\end{array}$ \\
\cline { 2 - 6 }
\end{tabular}} & La Pampa & 145.907 & 25.914 & 101.338 & 138.169 \\
\cline { 2 - 6 } & Neuquen & 109.703 & 14.517 & 28.866 & 69.640 \\
\cline { 2 - 6 } & Río Negro & 196.695 & 9.241 & 42.242 & 128.190 \\
\cline { 2 - 6 } & Shubut & 242.039 & 3.748 & 23.065 & 80.330 \\
\cline { 2 - 6 } & Tierra del & 282.750 & 1.058 & 9.948 & 17.580 \\
\hline & Fuego & 21.499 & 477 & 2.504 & 2.190 \\
\hline & SubTotal & $\mathbf{1 . 3 6 2 . 3 5 9}$ & $\mathbf{1 0 3 . 3 6 9}$ & $\mathbf{3 2 9 . 5 6 8}$ & $\mathbf{9 3 1 . 8 1 3}$ \\
\hline & Resto del país & 1.523 .261 & 3.941 .542 & 8.082 .462 & a) \\
\hline & ToTAL & 2.885 .620 & 4.044 .911 & 8.412 .030 & b) \\
\hline
\end{tabular}

En términos políticos, los territorios pampeano-patagónicos inicialmente fueron agrupados bajo una única gobernación. Pero en 1884, el Congreso Nacional cambió de opinión y decidió subdividir el espacio, creando los Territorios Nacionales de La Pampa, Neuquén, Río Negro, Chubut, Santa Cruz y

6 Fuentes: Segundo Censo de la República Argentina, Mayo 10 de 1895, Buenos Aires, Talleres Gráficos de la Penitenciaría Nacional, 1898, Tomo: Población, BELTRÁn, J. (1917), Geografia de la Argentina, fisica, política y económica, Buenos Aires, Cabaut y Cía, p. 187; Tercer Censo Nacional, 1 de junio de 1914, Buenos Aires, Talleres Gráficos de L.J. Rosso y Cía, 1916, T. I a X. *Holmberg, E. (1900), Viaje por la Gobernación de los Andes (Provincia de Atacama), Buenos Aires, Imprenta de la Nación. **Bunge, A. (1984), Una nueva Argentina, Buenos Aires, Hyspamérica. a) y b): No se consideran los totales y subtotales de población para 1936 puesto que Bunge toma la fecha de 1938 y no la de 1936, como en el resto de las jurisdicciones. El total era de 12761000 personas para 1938. 
Tierra del Fuego (similar política se siguió con los espacios del noreste del país, Chaco y Formosa, arrebatados a otros grupos indígenas, y con Misiones y Los Andes). El gobernador de cada uno de esos Territorios Nacionales era nombrado directamente por el Poder Ejecutivo, a diferencia de las viejas provincias, que gozaban de la autonomía y el autogobierno concedidos por una constitución de espíritu federal, dictada en 1853. En consecuencia, para muchos contemporáneos, los Territorios Nacionales funcionaron como una suerte de «colonias internas», gobernadas desde la capital. Quienes allí vivían no podían elegir autoridades (salvo municipales) ni enviar representantes al parlamento: su perfil se asemeja más al del habitante que al del ciudadano. En ese sentido, podemos señalar que el Estado nacional era la única presencia pública en la región, puesto que los agentes dependientes de los erarios fiscales municipales eran mínimos.

Pero, ¿qué era el Estado en el sur de Argentina entre 1880 y 1940? La caracterización del sector público en ese área es necesariamente paradójica, puesto que presenta dos perfiles difíciles de compatibilizar: a) por un lado hay que recordar que el Estado constituye el actor más importante de la vida pampeano-patagónica prácticamente a lo largo de todo el siglo XX. Protagonismo de múltiples dimensiones: en la configuración de identidades, pero también político, económico y social ${ }^{7}$. En ese sentido, en estas tierras no hay una sociedad previa al Estado nacional: éste es el que procura regularla y darle forma después del sometimiento de las fuerzas indígenas. No hay grupos sociales preestatales que sobrevivan con la capacidad como para imponerle condiciones de negociación al Estado y sus proyectos; b) por el otro lado, también es menester señalar que el Estado en los Territorios del Sur es, medido en términos materiales y de capacidad de regulación, muy pobre. La planilla de sueldos de las gobernaciones evidencia que el grueso del presupuesto anual se destinaba a sostener el cuerpo policial. Ese apartado consumía sumas notoriamente superiores a las que tenían otros destinos. La inversión en educación, infraestructura, salud y otros rubros era muy baja y no era un dato menor el que las partidas correspondientes a los gastos generales y salarios llegaran con varios meses de re-

7 Aranciaga, I. (2004), Representaciones de la nación en Patagonia. En VerniK, E. (comp.), Qué es una nación. La pregunta de Renan revisitada, Buenos Aires, Prometeo, pp. 97-11; CABRAL MÁRQues, D. (2003), La intervención del Estado en los procesos de construcción de las identidades socioculturales en la Patagonia Austral, Espacios, 26, 182-212, especialmente p. 198; NAVArro Floria, P. (1999), Historia de la Patagonia, Buenos Aires, Ciudad Argentina, p. 194. 
traso y obligaran a los empleados públicos y agentes a vincularse con comerciantes y hacendados que les adelantaran su sueldo ${ }^{8}$.

La demanda que se repite una y otra vez por parte de los gobernadores es el incremento del presupuesto público que recibían sus administraciones. El panorama de pobreza material que había denunciado en 1917 el comisario Gómez se repite ad infinitum en los archivos pampeanos y patagónicos. A la solicitud que hacían las autoridades se le sumaban las quejas de la prensa local y los ecos de ésta en Buenos Aires, en las que se daba a conocer el pasmoso estado de pobreza del Estado y la inutilidad de sus intervenciones públicas en educación, seguridad y salud. ${ }^{9}$ La Memoria de la gobernación de Río Negro sentenciaba en 1932 que era «vergonzoso y miserable el mobiliario de algunas dependencias formado por cajones de nafta como mesas y armarios» ${ }^{10}$.

Hay un elemento que ilustra claramente la debilidad de la presencia pública en la Patagonia. Sabemos que el Estado en los Territorios Nacionales no se apropió (ni siquiera intentó hacerlo) de potestades de la Iglesia católica o de asociaciones intermedias o étnicas para atender la formación de los niños, lo cual ha sido descubierto para regiones centrales del país, y que forma parte de un proceso continental más amplio. De hecho, la orden salesiana suplantó al Estado nacional en la provisión de servicios de salud y de educación en el sur hasta - por lo menos - el segundo tercio del siglo XX, exactamente en el mismo período en que se le arrebataban a la Iglesia sus potestades exclusivas para asentar nacimientos, matrimonios y defunciones. Junto con las tropas al mando del general Roca, ingresó en 1879 al territorio patagónico la orden de los salesianos: éstos, tuvieron mucha más fortuna que los jesuitas en siglos anteriores, y establecieron desde 1875 una red de iglesias y colegios, que resultó, en el largo plazo, hegemónica ${ }^{11}$. La estructura edilicia con la que contaban los salesianos, su organización de los recursos humanos y una innegable vocación misionera los convirtieron en gestores de la educación de amplios sectores de la población patagónica ${ }^{12}$.

8 Bohoslavsky, E. (2007), La policía de Neuquén (1884-1946). Una mirada a la institución desde sus agentes y oficiales, ponencia presentada en las II Jornadas de Historia Social de la Patagonia, Neuquén.

9 Un diario de circulación en todo el país comentaba muy negativamente el estado de la policía en el Territorio Nacional de La Pampa: «se comenta en términos desfavorables el abandono en que la superioridad tiene a la policía en lo referente al armamento. Se usan carabinas rémington completamente anticuadas, de un solo tiro, que tienen en su mayoría la rara propiedad de funcionar mal» (La Nación, 13 diciembre 1921, p. 10).

10 Sarobe, J.M. (1935), La Patagonia y sus problemas, Buenos Aires, p. 337.

11 NAVARRO FlORIA (1999), pp. 114-119.

12 BAndieri, S. (2005), Historia de la Patagonia, Buenos Aires, Sudamericana, p. 172. 


\section{SALUD Y ENFERMEDAD EN EL SUR ARGENTINO}

Para el Estado nacional, la salud de los territorios del sur argentino no fue un problema central hasta los inicios del siglo XX, y aún entonces, su ingreso en la agenda pública se hizo a través de organismos con escaso presupuesto y más escaso personal. Esta marcada desatención se pretendía excusar recurriendo a un imaginario salutífero, que destacaba como beneficiosos ciertos rasgos de los amplios espacios pampeano-patagónicos. Éstos, se decía, tenían una doble particularidad que los blindaba contra las epidemias: su población era en su mayoría joven y sana pues no sufría los encierros generados por las urbes y su clima, de vientos fríos y secos, barrería periódicamente las enfermedades pestilentes. Como se verá, esta argumentación poco tenía que ver con la situación concreta de los pobladores, alejados de los centros médicos y de las tendencias nacionales de medicalización de la vida personal, familiar y comunitaria.

La intervención del Estado en la salud pública se daba principalmente en la ciudad de Buenos Aires y en la región litoral del país. Allí, tal como ha mostrado la literatura, la construcción de los centros hospitalarios y carcelarios en fechas cercanas al Centenario fue la expresión de la «máquina perfecta» para separar, curar y regenerar a partir del control médico-penitenciario. Los edificios fueron el ejemplo de una nueva estética, la del higienismo: amplios, luminosos, limpios, construidos con nuevos materiales, reduciendo sus componentes a las funciones requeridas. La Penitenciaría Nacional, el Hospital de Clínicas o el Italiano (todos en Buenos Aires), así como la Cárcel de Reincidentes de Ushuaia (Territorio de Tierra del Fuego) y los grandes centros sanitarios promovidos por la Ley 4953, muchos de ellos para los Territorios Nacionales, se planificaron en un amplio espacio y con la lógica del panóptico ${ }^{13}$.

De los hospitales proyectados por esa legislación - y publicitados en la obra de Domingo Cabred, director de la Comisión de Asilos y Hospitales Regionales ${ }^{14}$ - conviene realizar algunas precisiones: El Hospital Regional de Río Negro, en Allen, se inauguró hacia 1925 y el de la Pampa, en Santa Rosa, recién en 1938, veinte años después del anuncio oficial. En un discurso

13 LiEnUR, J.F., (2000), La construcción del país urbano. En LobATo, M.Z. (dir.), Nueva Historia Argentina. El progreso, la modernización y sus límites, Buenos Aires, Sudamericana, pp. 450-451.

14 CABRED, D. (1918), Discursos pronunciados con motivo de la colocación de la piedra fundamental de los asilos y hospitales regionales en la República Argentina (Ley 4953), Buenos Aires, Talleres Gráficos J. Weiss y Preusche. 
inaugural, el funcionario realizó un prolijo recuento de los problemas sanitarios de los Territorios. Se especificaban así las notables diferencias entre los dos países que comprendían la Argentina de entonces: en Buenos Aires había 3,19 camas por cada 1000 habitantes, mientras que las 309 camas instaladas en todos los Territorios Nacionales arrojaban un promedio de 0,93 . La mortalidad era en 1899 de 24,80/1000 para los territorios patagónicos y 17,4/1000 en Buenos Aires.

Hasta ese momento, también fue una utopía el establecimiento de centros de salud. En los Territorios, los pequeños hospitales y clínicas existentes sobrevivían con subsidios municipales y el esfuerzo de las pequeñas comunidades. Fueron miembros de familias notables locales (en la Pampa fueron siempre mujeres, atendiendo a una demanda social de género) o bien policías y las órdenes religiosas (salesianos, en gran parte de la Patagonia) los que organizaron modestas salas de primeros auxilios y hospitales sin complejidad técnica para la atención de los más indigentes. Para las enfermedades contagiosas y frente a casos de inusitada virulencia - epidemias de cólera, viruela y de peste bubónica - se organizaron ocasionalmente «casas de aislamiento», pero esos hospitales resolvían sólo los casos de brotes agudos y los problemas más graves de salud, y dejaban de tener sostén municipal o nacional una vez culminado el peligro.

El Estado nacional participaba de manera indirecta en esos casos, otorgando subsidios a las asociaciones benéficas a partir de lo recaudado por la Lotería nacional, pero los funcionarios territorianos observaban que la distribución no se hacía de manera equitativa: las instituciones que dependían de esos recursos para funcionar resultaban en desigualdad de condiciones frente a las de la Capital Federal y las provincias. Tal era el caso de los pequeños hospitales, organizados por las sociedades de beneficencia locales, muchas veces financiados por donaciones de los notables por aportes de la comunidad y por la municipalidad, que ansiaban formar parte de las instituciones más favorecidas por los subsidios a nivel nacional.

En los Territorios de la Pampa, Río Negro y Neuquén escaseaban, además de los centros de salud, los médicos y otros técnicos sanitarios. En la primera década del siglo XX, no había más de uno o dos médicos por jurisdicción, ya que muchos profesionales preferían las posibilidades económicas o de perfeccionamiento brindadas en las urbes del litoral argentino. El Estado creó una figura para los Territorios, el «médico de la gobernación»; durante años se trató del único facultativo de toda una región con habilitación para funciones oficiales muy diversas y amplias. Era nombrado por el Departamento Nacional de Higiene y se lo consultaba para que actuase como perito legal. Ejercía 
también el rol de médico escolar, de la policía, de la cárcel y, si era preciso, del destacamento militar o de la subprefectura marítima, realizaba también la revisión sanitaria en las áreas urbanas y era consultado frente a posibles epidemias en todo el territorio a su cargo. Las variopintas y amplias funciones nos permiten reflexionar sobre su cumplimiento, que los mismos profesionales veían difícilmente realizables ${ }^{15}$. En ese marco, se comprende la supervivencia de prácticas curativas tradicionales en contextos urbanos y rurales.

En 1913, el Departamento Nacional de Higiene, máximo organismo nacional en la aplicación de políticas sanitarias, resolvió solucionar en parte las carencias de los Territorios Nacionales, fundando Asistencias Públicas. Estas instituciones tenían una serie amplia de funciones y un área enorme a cubrir para tareas de vacunación, vigilancia epidemiológica, medicina legal y otras muchas más. En los tres Territorios analizados, estuvieron a cargo de escaso personal (generalmente un médico y dos técnicos) y podían hacer sentir la influencia en las capitales (Santa Rosa, Neuquén y Viedma), pero más allá de los pequeños poblados, su recepción era escasa.

Hasta 1930, salvo la excepción del Hospital Regional de Allen (Río Negro), no se organizaron otros centros de atención. Los reclamos fueron constantes y la transformación de esta política de abandono sanitario se debió a la percepción entre médicos y gobernantes de distinto signo político - tanto de la izquierda como de la derecha - del avance de enfermedades sociales (como la tuberculosis y la sífilis). Ante una visión negativa del potencial generativo del país, aumentó el impacto de las instituciones vinculadas con la atención de tales dolencias y florecieron en Buenos Aires y las principales ciudades del Litoral una red de instituciones dedicadas con exclusividad a la atención materno-infantil. En los Territorios, se organizaron también centros maternales oficiales - dependientes de las Asistencias Públicas-, con hogares para niños y atención externa de visitadoras y otro personal especializado ${ }^{16}$.

15 Ver Anales del Departamento Nacional de Higiene, años: 1900, 1901, 1905, 1908 y 1911. Sobre los relatos médicos, consultar Di LISCIA, M.S. (e.p.), Imaginarios y derroteros de la salud en el interior argentino. Los Territorios Nacionales (fines del XIX y principios del

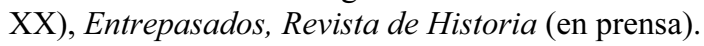

16 El de La Pampa data de 1930, pero a finales de esa década recibió financiamiento estatal, durante años dependió de la Sociedad de Beneficencia y tenía personal ad honorem. Los de Neuquén y Río Negro fueron fundados en 1938 y 1939, respectivamente (Di LiSCIA, M.S. (2007), Instituciones, médicos y sociedad. Las posibilidades y los problemas en el Territorio pampeano (1884-1933). En Di Liscia, M.S., Lluch, A. y Lasalle, A. (ed.), Al oeste del paraíso. La transformación del espacio natural, económico y social en la Pampa Central (Siglos XIX-XX), Buenos Aires, Miño y Dávila-EDULPam, pp. 123-154 y Olaván Chaus, A. 
Hacia 1940, el primer registro sanitario institucional de todos los Territorios - que hasta ese momento había sido parcial y fragmentado-, concluía revelando que había 409 médicos y 1494 camas para todos los Territorios,

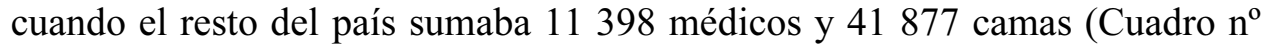
2). Si consideramos la población existente en 1936 en los Territorios y en 1938 para todo el país ${ }^{17}$, el total de médicos por habitantes era para todo el país de 8,93, mientras que en los Territorios llegaba a 4,39. La relación entre cantidad de camas/habitantes era 0,003 en el país, pero en los Territorios no pasaba de 0,001. En los tres Territorios que nos atañen especialmente las cifras variaban: Neuquén tenía por entonces 2,15 médicos/habitantes y 0,001 camas/habitantes; Río Negro 3, 90 médicos/habitantes e igual proporción de camas. La Pampa estaba por encima de la media de los Territorios en uno y otro rubro: 6,44 médicos/habitantes y 0,002 camas/habitantes. A pesar de estas diferencias, lo que las cifras indican es una debilidad institucional muy marcada en estas áreas del interior argentino respecto a las posibilidades para un proceso exitoso de medicalización, aún a finales del período analizado.

Hacia 1945, Aquiles Ygobone marcaba con acento desesperado la multiplicidad de problemas sanitarios y sociales de los territorios del Sur, en clave de discriminación regional: «Frente a los suntuosos nosocomios metropolitanos y al prestigio y nutrido cuerpo médico de los sanatorios e institutos sanitarios de las capitales de provincia», denunciaba «el abandono en que yacen los enfermos y la población de dilatadísimas zonas del sur del país». Este investigador apelaba —nuevamente - a la «acción profiláctica» del clima como única explicación de la limitación de los focos epidémicos, pero clamaba por una intervención estatal, para resolver «las causas predisponentes de las enfermedades: descendencia o herencia alcohólica, debilidad fisiológica de los hijos de los aborígenes y la consanguinidad», en áreas con población inmigrante. El sarampión, el coqueluche y las enfermedades pulmonares graves, entre otras tantas, afectaban cada vez más a la dispersa población de la Patagonia. Y agregaba que la mayoría de estas víctimas desamparadas y analfabetas sufría desnutrición desde la infancia, y en su madurez frecuentaba la

(1940), Dirección de Maternidad e Infancia, Anales del Departamento Nacional de Higiene, 4 (1), 89-95, pp. 92-95).

17 Ver la aclaración del cuadro $n^{0} 1$. Por causas cuya explicación excede este trabajo, Argentina no tuvo censos generales entre 1914 y 1947, años en que se realizaron el III y IV censo nacional respectivamente. En los períodos intercensales, se realizaron estimaciones sobre la población. Sobre el particular, ver OTERO, H. (2006), Estadística y nación. Una historia conceptual del pensamiento censal de la Argentina moderna, 1869-1914, Buenos Aires, Prometeo. 
cárcel por robo, hurto o lesiones ${ }^{18}$. La conexión entre falta de atención sanitaria en la niñez y problemas de adaptación social posteriores era parte de una argumentación ya tradicional en los años cuarenta, pero su focalización en el interior argentino sí era una novedad.

CUADRO N ${ }^{\mathrm{o}}$ 2. INSTITUCIONES Y PROFESIONALES SANITARIOS EN LOS TERRITORIOS NACIONALES, $1940^{19}$

\begin{tabular}{|c|c|c|c|c|c|c|c|c|c|}
\hline \multirow{2}{*}{ Distritos } & \multirow{2}{*}{$\begin{array}{c}\mathrm{N}^{\circ} \text { de } \\
\text { médicos }\end{array}$} & \multicolumn{3}{|c|}{ Hospitales } & \multirow{2}{*}{ Dispensarios } & \multirow{2}{*}{$\begin{array}{c}\text { Salas } \\
\text { Primeros } \\
\text { auxilios }\end{array}$} & \multirow{2}{*}{$\begin{array}{l}\text { Asistencia } \\
\text { pública }\end{array}$} & \multirow{2}{*}{$\begin{array}{c}\text { Total } \\
\text { Servicios } \\
\text { Sanitarios }\end{array}$} & \multirow{2}{*}{$\begin{array}{l}\mathrm{N}^{\circ} \text { de } \\
\text { camas }\end{array}$} \\
\hline & & 1 & 2 & 3 & & & & & \\
\hline Misiones & 39 & 2 & 1 & 4 & 8 & 1 & 1 & 17 & 230 \\
\hline Chaco & 82 & 2 & 2 & 1 & 5 & 10 & 1 & 20 & 267 \\
\hline Formosa & 15 & - & - & 1 & 1 & 1 & 1 & 4 & 105 \\
\hline Los Andes & 3 & - & - & - & - & - & - & - & - \\
\hline Río Negro & 50 & 1 & 1 & 2 & 1 & 6 & 1 & 12 & 196 \\
\hline La Pampa & 89 & 1 & 2 & 7 & - & 3 & 1 & 14 & 380 \\
\hline Neuquen & 15 & 3 & - & - & - & 1 & 3 & 7 & 71 \\
\hline Santa Cruz & 23 & - & 2 & - & 1 & 2 & 1 & 6 & 43 \\
\hline Chubut & 90 & 1 & 1 & 1 & - & 3 & 3 & 12 & 202 \\
\hline T. del Fuego & 3 & - & - & - & - & - & 1 & 1 & - \\
\hline Total TN & 409 & 10 & 9 & 16 & 16 & 27 & 13 & 93 & 1.494 \\
\hline Resto país & 11.398 & 32 & 105 & 254 & 277 & 115 & - & 780 & 41.877 \\
\hline TOTAL & 11.807 & 42 & 114 & 270 & 293 & 142 & 13 & 873 & 43.371 \\
\hline
\end{tabular}

\section{DEFINIR, SEPARAR Y CURAR LA ANORMALIDAD}

Como ha subrayado con eficacia la historiografía, el sistema educativo y el sistema policial y carcelario utilizaron el discurso positivista de la peligrosidad y la anormalidad para gestar marcas diferenciadoras entre la población, que implicaron una subordinación de etnia, género, nacionalidad y clase ${ }^{20}$. En

18 Ygobone, A. (1945), La Patagonia en la realidad argentina. Estudio de los problemas sociales, económicos e institucionales de las gobernaciones del Sur, Buenos Aires, El Ateneo, pp. 123-124.

19 Fuente: Boletín Sanitario, DNH, año IV, 1940. 1: Hospitales nacionales, 2: Hospitales municipales, 3: Hospitales de beneficencia y particulares (solo en TN) y Hospitales provinciales y de colectividades (resto del país).

20 CAIMARI, L. (2004), Apenas un delincuente: crimen, castigo y cultura en la Argentina, 1880-1955, Buenos Aires, Siglo Veintiuno Editores; Ruggiero, K. (2003), Sexual aberration, degeneration and psychiatry in Late-nineteenth-Century Buenos Aires. En Plotkin, M. (ed)., Argentine on the Couch. Psychiatry, State and Society 1880 to the Present, Albuquerque, University of Mexico Press, pp. 49-84 y Ruggiero (2004), Modernity in the Flesh. Medicine, 
los Territorios, la difusión de las prácticas, sin embargo, estaba lejos de las expectativas de los juristas, psiquiatras, médicos, criminalistas y otros especialistas, para quienes moralidad e higiene se unían «naturalmente» en la conformación de ciudadanos y mujeres obedientes y trabajadores ${ }^{21}$. Los sujetos peligrosos sobre los cuales era preciso el ejercicio de regeneración en realidad eran un conjunto informe y difícil de definir, donde se alojaban tradiciones o resistencias a las pautas capitalistas y a las normas burguesas: prostitutas, anarquistas o simplemente, trabajadores descontentos, desempleados, vagabundos, discapacitados y enfermos.

De acuerdo a las enunciaciones positivistas, la separación de la normalidad de la anormalidad se debía producir ya desde las escuelas. El temor por la irrupción de una inmigración descontrolada, que pudiese infectar la pureza con los «vómitos» de otros pueblos y contagiar así la sangre pura de la nacionalidad argentina estuvo en las gargantas de los pedagogos. Los Territorios constituían uno de los eslabones más débiles, puesto que allí, supuestamente, la población nueva no modificaba sus costumbres extranjeras sino que lograba reproducirlas ante la ausencia de una vigilante mirada estatal. Con encendido discurso, un inspector docente decía en 1910:

«La nación no ha podido llevar a ellos más que su administración y no el influjo de su civilización. Un cuerpo de leyes y reglamento, una policía fraccionada en unidades, un cierto número de jueces y empleados no son el mejor instrumento (...) la misión que no pudo llevar la administración nacional, por falta de medios, tiene que cumplirla la escuela» ${ }^{22}$.

Desde finales del siglo XIX, se gestó en las instituciones educativas un servicio médico escolar, cuya primera función fue detectar focos epidémicos y controlar al personal docente, alumnos y establecimientos para impedir el avance de la peste, la fiebre amarilla, el cólera, la viruela, el sarampión o, avanzado el XX, la difteria y la tuberculosis. Su organizador, Emilio Coni,

Law and Society in Turn-of-the Century Argentine, Stanford, Stanford University Press; CARLI, S. (2002), Niñez, pedagogía y política. Transformaciones de los discursos acerca de la infancia de la educación argentina entre 1880-1955, Buenos Aires, Miño y Dávila.

21 A principios del siglo XX, Eusebio Gómez, uno de los mayores especialistas sobre penitenciaría en el país, expresaba que los Territorios carecían, salvo el de Tierra del Fuego, de instituciones adecuadas para el tratamiento científico, donde los internos tuviesen «disciplina, educación y trabajo», GoMEZ, E. (1912), El problema penal argentino, Archivos de Psiquiatría, criminología y ciencias afines, tomo XI, año 1912, 394-432, especialmente p. 421.

22 Ramos, J. (1910), Historia de la Instrucción primaria de la República Argentina, Buenos Aires, T. I, p. 600. 
intentó que, desde su ingreso al sistema, los niños estuviesen vacunados contra la viruela, único medio eficaz contra la dolencia y cuya obligatoriedad existía desde 1886. Con la llegada a la dirección del Consejo Nacional de Educación de José María Ramos Mejía, reconocido psiquiatra, el Servicio Médico Escolar recibió mayor impulso, y en la primera década del siglo XX, la influencia positivista se dejó sentir en la formación de escuelas de niños débiles y en el fortalecimiento de una perspectiva médica francamente discriminatoria, para la observación de los escolares. Los listados de niños apáticos, retardados, débiles o coléricos, con patologías mentales o incapacidades físicas (problemas de visión, de oído, desnutrición, etc.) que aparecen en las fuentes de entonces implicaba la realización de las complicadas pruebas indicadas por la antropología física de moda para demarcar los «cuerpos degenerados» de los más sanos y aptos ${ }^{23}$.

De acuerdo a la documentación oficial, los maestros se entusiasmaron con las técnicas positivistas más modernas y las aplicaron profusamente, sobre todo en la Capital Federal. Nos permitimos dudar sobre el impacto de mediciones de cráneo y de la aplicación de las encuestas sobre los antecedentes hereditarios, entre otras técnicas para la formación de un «biotipo» argentino, fuera de las ciudades más importantes de Argentina ${ }^{24}$. En el interior y sobre todo en los Territorios, el impacto médico se produjo a partir de una serie de pautas higiénicas, basada en la sugestión más que en la imposición. El tinte de moralidad que éstas implicaban (el mensaje principal apuntaba a la necesidad de «ser limpio y ser bueno»), no debe oscurecer su eficacia para la resolución de los problemas de salud.

Por diversas razones - escasez de médicos, valoración de los docentes como «héroes» de la nacionalidad y la modernidad - los maestros se convirtieron en divulgadores y practicantes del mensaje científico, cuya expansión material estaba desajustada, como vimos en el apartado anterior, con las posibilidades efectivas de realización. La escasez de instituciones y técnicos, así como un bajo presupuesto, conspiraban contra la aplicación de políticas de «normalización» o medicalización compulsiva. La respuesta fue intensificar la tarea docente, extendiendo así parte de la labor médica. Específicamente, el

23 Informe de la Inspección General de Territorios, 1911, 1912, 1913, Buenos Aires, Consejo Nacional de Educación, 1914.

24 Di LisCiA, M.S. (2008), Los bordes y límites de la eugenesia, por donde caen las 'razas superiones' (Argentina, primera mitad del siglo XX), en: Miranda, M. y VALLEJo, G. (eds.), Politicas del cuerpo. Estrategias modernas de normalización del individuo y la sociedad, Buenos Aires, Siglo XXI, pp. 377-409. 
Consejo Nacional de Educación preveía que, en los Territorios Nacionales, fuesen maestros los encargados de diagnosticar los primeros problemas de salud e incluso de resolverlos: la profilaxis de epidemias, la vacunación, la detección de problemas de salud (vista, oído, enfermedades de la piel) y otra serie muy amplia de funciones se anexaron al reconocimiento de dificultades pedagógicas y adaptativas de los alumnos ${ }^{25}$. De esa manera, las instituciones escolares fueron, en los Territorios, una avanzada para la medicalización de la población: a partir de los alumnos, los maestros podían llegar al resto del grupo familiar e intensificar la propaganda higiénica. Esta forma de extensión del brazo regulador y sanitario del Estado puede ser vista como una opción innovadora, pero a la vez mucho más económica que implementar servicios de salud. Se trata también de una posibilidad que limita el alcance y la aplicación de los mismos principios higiénicos que proclama, en la medida en que deja en manos de un personal menos instruido una enorme responsabilidad (y cierto margen para la aplicación de los programas).

En el caso de las instituciones para enfermos mentales y criminales, los Territorios también estuvieron a la zaga en la conformación de ámbitos de puesta en práctica de los discursos positivistas sobre la regeneración social y científica. De acuerdo con las propuestas consensuadas entre un amplio conjunto de especialistas, el Estado debía gestar una red de instituciones para los «alienados, retardados, cretinos, alcoholistas, epilépticos, niños vagabundos», entre otros marginados ${ }^{26}$. Y a la vez, los asilos para enfermos mentales debían rehacerse como verdaderos hospitales y no prisiones ${ }^{27}$. Desde finales del siglo XIX, las insistencias de implementación de un sistema «racional» y científico para la separación y readaptación de los anormales fue problemática en el

25 En el «Proyecto de disposiciones generales adaptables a las necesidades de las escuelas de los Territorios Nacionales» se impulsaba la vacunación antivariólica y el examen diario de los alumnos, para detectar enfermedades infecciones, y «en la imposibilidad de que la tarea la realicen médicos inspectores, hemos pensado que podríamos recurrir a los maestros y directores de escuelas quienes, con la inteligencia y abnegación que les caracteriza y convenientemente ilustrados sobre los principales síntomas». También se daban instrucciones sobre desinfección de ropas y locales, y sobre la detección y control de la pediculosis e incluso, se imprimieron folletos ad hoc (El Monitor de Educación Común, CNE, 25 (395), 1905).

26 En el plan general de Cabred (1918), resulta sugestivo observar que sí se fundaron asilos para huérfanos, alienados y retardados en la Provincia de Buenos Aires (Asilo Colonia Regional Mixto de Retardados, en Torres, y Asilo Colonia Regional de Niños abandonados en Olivera).

27 Loudet, O. (1928), El médico de las prisiones, Anales del Instituto de Medicina Legal, Buenos Aires, T. I, 123-133, especialmente p. 124. Loudet era en ese momento Director del Instituto de Criminología, el más importante del país en relación con la investigación y práctica de la psiquiatría y criminología. 
interior del país. Los vecinos de las pequeñas localidades y la policía iniciaban el proceso, incluso, con argumentaciones de base médica y apuntando al control social de vagabundos, enfermos y discapacitados ${ }^{28}$. Así, las personas que circulaban por las extensas soledades de la Pampa y la Patagonia sin «oficio ni beneficio» teóricamente podían ser detenidas y si la situación concreta lo permitía, limitar sus derechos por causas médicas luego de un juicio por insanía.

Alcohólicos, psicóticos o neuróticos graves, discapacitados físicos y mentales eran incluidos en la «grilla demarcatoria» de los anormales, pero el señalamiento social de la peligrosidad era sólo el inicio del biopoder sobre los cuerpos. La regulación institucional constituía la clave para completar el ciclo y tal situación no se produjo: los centros de salud y las cárceles territorianas carecían de las tecnologías modernas de control social y de los técnicos para aplicarlas con suficiencia y capacidad. De acuerdo con las fuentes consultadas, no hubo lugares específicos de tratamiento para los insanos, quienes en los casos más graves, eran trasladados a los grandes centros de Buenos Aires (Hospicio de Las Mercedes, Hospital de Alienadas o Colonia-Hospital de Luján, también llamado «Open Door»).

Los registros alarmantes de los miembros de la Liga de Higiene Mental, muy vinculados al movimiento eugenista argentino, expresaban que los hospicios porteños estaban colapsados, pese a lo cual las personas que debían estar bajo tratamiento eran muchas más de las que estaban allí encerradas ${ }^{29}$. Al margen de estos análisis sesgados, cuya raíz estaba en las teorías degenera-

28 Para la Pampa, ver Di LiSCIA, M.S. (2003), Locura y peritaje médico legal. Acerca de la justicia civil en el interior argentino, 1890-1930, Estudios Sociales, XIII (25), 147-165 y Di LiSCIA, M.S. y BiLlorou, M.J. (2003), Locura y crimen en el discurso médico-jurídico. Argentina, Territorio Nacional de la Pampa, ca. 1900, Anuario de Estudios Americanos, 60 (2), 581-606.

29 El médico Arturo Ameghino señalaba que: «Se puede decir sin exageración que la asistencia social del alienado deja mucho que desear en nuestro país. Las plazas disponibles, que hace diez años eran ya notoriamente escasas, apenas aumentaron luego, y en cambio la población del país, que hace diez años no alcanzaba a 8 millones de habitantes, pasa ya de los $11 »$. En este y otros textos similares, es constante la denuncia de saturación de los espacios de atención, por ejemplo, se señalaba que en lugares donde caben 1000 alienados de hasta 2000 pacientes, muchos de ellos extranjeros y de fuera de la Capital Federal o de la Provincia de Buenos Aires. Ver AmEghino, A. (1927), Carácter y extensión de la locura en las diversas regiones de la República Argentina, Revista Argentina de Neurología, Psiquiatría y Medicina Legal, Vol. I, 494-504. Otros ejemplos en Rossi, A. (1940), Higiene mental y asistencia social, Anales de Biotipología, engenesia y medicina social, 5 (89), 49-52, quien mencionaba 50 000 pacientes sin tratamiento, una epidemia de «locos sueltos» en Buenos Aires, verdadera pesadilla para psicólogos y sociólogos. 
cionistas y la antropología física, la preocupación médica era la incapacidad total de atención de los pacientes del interior, tanto de provincias como de los Territorios. Aún en los años treinta, La Pampa carecía de espacios dedicados a la atención de los insanos y, a mediados de los cuarenta, era un reclamo pendiente para toda la Patagonia ${ }^{30}$. Entre 1909 y 1913, ingresaron 69 enfermos de La Pampa y respecto a toda la Patagonia, se mencionan, en un período posterior, treinta dementes derivados por año a los hospicios de Buenos Aires y Capital.

La única «atención» a los enfermos, en sus lugares de origen se brindaba, paradójicamente, en los calabozos. A pesar de tratarse de una situación denostada por los psiquiatras, los enfermos podían quedar días, semanas e incluso meses sin atención médica. Esta situación escandalosa, que observamos a principios de siglo, persistió en amplias áreas durante décadas y contradice una imagen de severo control social en todos los órdenes ${ }^{31}$. Lo mismo sucedía en el caso de internos aquejados de enfermedades mentales abandonados en las cárceles. $\mathrm{Si}$ consideramos que éstas no presentaban, bajo ningún punto de vista, establecimientos modelos en la atención de los presos comunes (carecían de talleres, de escuelas y de elementos de observación psiquiátrico-criminológica) ${ }^{32}$, en el caso de los enfermos mentales, se trataba de un agravante.

Una mirada a los ámbitos de encierro y supuestos espacios de la ortopedia social, las cárceles, muestra la más completa ausencia de desarrollo de espacios médicos dentro de las unidades de detención. En el caso de la cárcel de Neuquén, que albergó a lo largo de la primera mitad del siglo XX entre 200 y 500 presos, no hay registros de la constitución de salas de enfermería hasta la década de $1940^{33}$. En ese decenio se llevaron adelante una serie de reformas e

30 El director de la Asistencia Pública de Santa Rosa, Mario Cabella, señalaba estos aspectos en un Informe técnico (Nota dirigida al Gobernador de la Pampa, Exp. M.791/1933, Gobernación de la Pampa, MNR no 303 R, AHP, LP). Ver asimismo AMEGHINO (1927), pp. 500-501.

31 «No hay más atención que en los calabozos para estos pobres desamparados; en el mejor de los casos, se los traslada a Buenos Aires», donde ya están superpoblados los centros. No sería mejor, «más práctico, económico y humanitario, crear casas de salud en la Patagonia». YGOBONE (1945), p. 126.

32 Eran frecuentes, como señalamos en el primer apartado, las denuncias sobre hacinamiento de los internos y mal estado de las cárceles territorianas. Incluso, fue necesario en ocasiones cerrar completamente un establecimiento y trasladar a los presos a otro distrito, como sucedió en La Pampa, cuando los graves problemas edilicios de la cárcel de penados de General Acha requirieron el traslado de 23 presos a Neuquén durante un año (Diario La Autonomía, año II, 16-01-1917, AHP, LP).

33 Bohoslavsky, E. y Casullo, F. (2003), Sobre los límites del castigo en la Argentina periférica. La cárcel de Neuquén (1904-1945), Quinto Sol, 7, 37-59. 
inversiones en infraestructura de magnitud, que permiten alivianar en parte las muy malas condiciones de vida de la población carcelaria y del personal penitenciario. Ya en tiempos peronistas, la pequeña burocracia de la cárcel neuquina podía ofrecer orgullosamente los resultados cuantificados de las actividades médicas sobre la población indicando la cantidad de curaciones, radiografías e intervenciones quirúrgicas realizadas, entre otras prácticas. ${ }^{34}$.

Las autoridades centrales, que inspeccionaban periódicamente los Territorios, conocían las dificultades pero las soluciones tardaban en llegar. En los exhaustivos informes de 1924 y 1925 del Ministro de Justicia e Instrucción pública, Antonio Sagarna, sobre la tarea anual realizada por ese organismo, aparecen dos niveles diferentes: por un lado, la labor legislativa (cuya vedette era el proyecto de Ley de Estado Peligroso) y las investigaciones llevadas a cabo en el Instituto de Criminología, con los boletines médico-psicológicos que realizaba Eusebio Gómez, en la Penitenciaría Nacional. Por otro lado, los informes relativos a los distintos Territorios nacionales, donde se recogían la cantidad de internos en las cárceles de encausados y/o penados. En estos últimos casos, no se menciona algún tipo de observación médico-psiquiátrica, sino una estadística anual de los presos a la que se agregaban las enfermedades (tuberculosis, gastritis, las más frecuentes) y los delitos por los cuales se encontraban recluidos ${ }^{35}$. Esta divergencia es un ejemplo de la situación concreta de las instituciones del interior, huérfanas de la aplicación de las políticas científicas de control social.

\section{CONClusiones}

En 1913 fue inaugurada la Asistencia Pública en Trelew (Gobernación de Chubut), con la presencia de Luis Navarro, representante del Departamento Nacional de Higiene. En esa ocasión, Navarro reconoció la diferencia entre el accionar de la salud pública en Buenos Aires y el escuálido presupuesto que recibían los Territorios Nacionales: en la capital, donde vivía un millón de almas, expuso, la acción pública había eliminado el fantasma de la peste: allí, a pesar de la aglomeración, había más seguridad que en nuestras soledades,

34 Archivo histórico de la Provincia de Neuquén, Memoria del gobernador de Neuquén correspondiente al año $1949, \mathrm{f}^{\circ} 91$.

35 Sagarna, A. (1924), Memoria presentada al H. Congreso de la Nación por el Ministro de Justicia e Instrucción pública Dr. A. Sagarna, T. I, Buenos Aires, Talleres Gráficos de la Penitenciaría Nacional y SAgarna, A. (1925), Anexos a la Memoria presentada al H. Congreso de la Nación por el Ministro de Justicia e Instrucción pública Dr. A. Sagarna, T. I, Buenos Aires, Talleres Gráficos de la Penitenciaría Nacional. 
que no tienen más profilaxis que el viento ${ }^{36}$. En efecto, si alguna tendencia de largo plazo se puede descubrir en lo que se refiere a las políticas públicas de salud implementadas por el Estado nacional en el sur argentino, esa tendencia es el desinterés. Inversiones que no llegan, falta de presupuesto, ausencia de personal especializado, uso de docentes para que cumplan misiones médicas, hacinamiento, son algunas de las postales que es necesario obtener de este análisis. La contracara del desinterés público fue una intensa auto-organización de las poblaciones locales, que contribuyeron a la creación de salas de primeros auxilios, hospitales, escuelas, colonias de vacaciones y boticarios. Estas actividades se constituyeron como un ámbito en el que se fueron formando y consolidando figuras y grupos dirigentes que capitalizarían su experiencia en los tiempos de conversión de territorios nacionales a provincias ${ }^{37}$.

¿Resolvemos el estudio de las instituciones sanitarias en particular y de normalización en general, en el área pampeano-patagónica como simplemente un caso de atraso?, ¿alcanza con señalar que se trataba de un área del Estado nacional sub-financiada y desatendida o hay espacio para sugerir otra idea? Es claro que el Estado no enviaba en cantidad ni a tiempo las partidas necesarias para el sostenimiento de las instituciones sanitarias, lo cual obligaba a improvisar soluciones y estrategias alternativas, mucho menos «científicas», coherentes y eficientes de lo que las autoridades sanitarias nacionales deseaban. No hemos querido sostener que por esa razón, las áreas del sur argentino quedaron por fuera de las tendencias que la literatura ha encontrado para espacios centrales de Argentina: más bien postulamos que las diferencias que se podían hallar entre una y otras eran de orden material y presupuestario y no intelectual. Una revisión de los discursos producidos por los médicos que peritaban en la justicia patagónica, por ejemplo, da cuenta de que estos profesionales estaban muy actualizados en lo que se refiere a saberes médicos y tendencias contemporáneas y que seguían muy de cerca las discusiones que se llevaban a cabo. De hecho, resulta muy marcado el defasaje entre la posesión y uso de jerga ideológica-científica del que se servían buena parte de los miembros de aparatos policiales, judiciales y sanitarios asentados en La Pampa y Patagonia ${ }^{38}$ y

36 Anales del Departamento Nacional de Higiene, Buenos Aires, T. XX, 1913: 1180.

37 Cfr. en relación a La Pampa, Di LisCiA, M.S. (2007). Sobre el problema de la formación de la dirigencia en la norpatagonia, y su vinculación con instituciones públicas y la prensa, cfr. PRISLEI, L. (2001), Pasiones sureñas: prensa, cultura y política en la frontera norpatagónica, 1884-1946, Buenos Aires, Prometeo.

38 Salgado, L. y AzAR, P. (2000), Evolucionismo y delincuencia: Los fundamentos biológicos de la Antropología Criminal, ponencia presentada en las I Jornadas de Historia del 
la dolorosa precariedad material que enmarcaba sus tareas cotidianas y pretensiones reformadoras y normalizadoras ${ }^{39}$.

Fecha de recepción: 24 de julio de 2008.

Fecha de aceptación: 26 de febrero de 2008.

Delito en la Patagonia, General Roca, 8 y 9 de junio; Salgado, L. y LizÁRraga, F. (2000), Patagónicos y lombrosianos, Ciencia hoy, 10 (59), 52-57.

39 Casullo, F. (2007), Administración de Justicia en el Territorio Nacional de Río Negro 1884-1934 y BoHOSLAVSKY, E. (2007), El brazo armado de la improvisación. Aportes para una sociología de los policías patagónicos 1880-1945, ambas presentadas como ponencias en el Seminario por una Historia de las Instituciones Estatales, Universidad Nacional de General Sarmiento, Los Polvorines, 17 y 18 de mayo. 